ITP-UH 24-95

hep-th/9509062

September 1995

\title{
Massive spinning particle on anti-de Sitter space
}

\author{
S.M. Kuzenko中: \\ Institut für Theoretische Physik, Universität Hannover \\ Appelstr. 2, 30167 Hannover, Germany

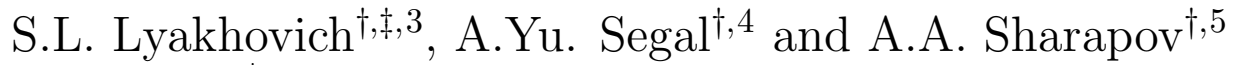 \\ $\dagger$ Department of Physics, Tomsk State University \\ Tomsk 634050, Russia \\ $\doteqdot$ Physics Department, Queen Mary and Westfield College \\ Mile End Road, London E1 4NS, UK
}

\begin{abstract}
To describe a massive particle with fixed, but arbitrary, spin on $d=4$ anti-de Sitter space $M^{4}$, we propose the point-particle model with configuration space $\mathcal{M}^{6}=M^{4} \times S^{2}$, where the sphere $S^{2}$ corresponds to the spin degrees of freedom. The model possesses two gauge symmetries expressing strong conservation of the phase-space counterparts of the second- and fourth-order Casimir operators for $s o(3,2)$. We prove that the requirement of energy to have a global positive minimum $E_{o}$ over the configuration space is equivalent to the relation $E_{o}>s, s$ being the particle's spin, what presents the classical counterpart of the quantum massive condition. States with the minimal energy are studied in detail. The model is shown to be exactly solvable. It can be straightforwardly generalized to describe a spinning particle on $d$-dimensional anti-de Sitter space $M^{d}$, with $\mathcal{M}^{2(d-1)}=M^{d} \times S^{(d-2)}$ the corresponding configuration space.
\end{abstract}

\footnotetext{
1 On leave of absence from: Department of Physics, Tomsk State University, Tomsk 634050, Russia (address after January 1, 1996).

${ }^{2}$ E-mail: kuzenko@itp.uni-hannover.de.

${ }^{3}$ E-mail: sll@phys.tsu.tomsk.su.

${ }^{4}$ E-mail: segal@phys.tsu.tomsk.su.

${ }^{5}$ E-mail: sharapov@phys.tsu.tomsk.su.
} 


\section{Introduction}

Not long ago, there were constructed twistor formulations for the massless BrinkSchwarz superparticle in dimensions $d=3,4,6$ and 10 [1,2] and later [3] for the heterotic $d=10$ Green-Schwarz superstring, which possess manifest invariance under both target-space supersymmetry and a world-line (world-sheet) general covariance supergroup and provide a geometric origion for Siegel's $\kappa$-symmetry. A central point in setting up these formulations was the use of bosonic twistor-like variables parametrizing the sphere $S^{d-2}$ regarded as a homogeneous space of the $d$-dimensional Lorentz group $[4,5]$.

In a recent paper [6], we proposed the model for a massive particle of arbitrary spin in $d=4$ Minkowski space $\mathbf{R}^{3,1}$ as a Poincaré-invariant dynamical system on $\mathbf{R}^{3,1} \times S^{2}$, where $S^{2}$ is the space of spin degrees of freedom. The model is based on simple physical and geometrical principles. Its quantization leads to the unitary massive representations of the Poincaré group. The physical wave-functions for particles of all integer spins span the space of on-shell scalar fields on $\mathbf{R}^{3,1} \times S^{2}$. For a given spin, the wave-functions form an eigenspace of a relativistic spherical Laplacian. Our model admits natural higher-dimensional and supersymmetric generalizations [7], with $\mathbf{R}^{d-1,1} \times S^{d-2}$ being the bosonic part of the configuration space.

The results of Refs. [1-3] and [6,7] indicate that extended space-times of the form

$$
d \text {-dimensional space-time } \times S^{d-2}
$$

deserve serious study. Their use may be relevant not only in the superstring context but also for constructing a consistent theory of higher-spin fields. It is worth noting that $\mathbf{R}^{d-1,1} \times S^{d-2}$ is the minimal homogeneous space of the $d$-dimensional Poincaré group, which contains the Minkowski space as a submanifold. In a curved space, on the other hand, the local Lorentz group can be naturally identified with a localized version of the conformal group of $S^{d-2}$. Manifolds of the above structure arise most simply in the framework of a massless spinless dynamics [2]. Below we are going to show the relevance of such manifolds for describing a massive spin dynamics in the case of space-times with constant curvature.

In the present paper, we generalize the model of Ref. [6] to the case of $d=4$ antide Sitter (AdS) space and discuss higher-dimensional extensions. The AdS space is known to be a maximally symmetric solution to the Einstein vacuum equations with a negative cosmological term (see, e.g., [8]). There are three basic grounds in favour of the AdS space as compared to the de Sitter space (positive cosmological constant). (i) The symmetry algebra of the AdS space, so(3,2), has unitary representations with bounded energy [9-11]. The positive-energy irreps, denoted $D\left(E_{o}, s\right)$, are classified by minimal energy and $E_{o}$ and spin $s, s=0, \frac{1}{2}, 1, \ldots$, with $E_{o}$ restricted by unitarity as follows (in dimensionless units for energy):

$$
E_{o} \geq s+\frac{1}{2}, s=0, \frac{1}{2} \quad E_{o} \geq s+1, s \geq 1 .
$$


$D\left(\frac{1}{2}, 0\right)$ and $D\left(1, \frac{1}{2}\right)$ correspond to the Dirac singletons [12], the irreps $D(2,0)$ and $D(s+1, s)$, for any $s$, describe massless particles [11,13]; finally, massive particles are associated with the choice $E_{o}>s+1$. (ii) Similarly to Minkowski space, the AdS space can be supersymmetrized. It presents itself the even part of AdS superspace $[14,15]$ that originates as a maximally symmetric solution of the superfield dynamical equatons in $N=1, d=4$ minimal supergravity with a cosmological term (see, e.g., $[16,17])$. (iii) This is the AdS space, neither flat nor de Sitter ones, that can arise as a classical vacuum solution in consistent theories of higher-spin massless fields including gravity $[18,19]$.

To describe a spinning particle on the AdS space denoted below $M^{4}$, we consider manifold $\mathcal{M}^{6}=M^{4} \times S^{2}$ as the configuration space. $\mathcal{M}^{6}$ turns out to be a coset space of the AdS group, i.e. the symmetry group of $M^{4}$. The chief dynamical principle underlying our model is the requirement of strong conservation for classical phasespace counterparts of the Casimir operators of the AdS group. This principle leads to unique gauge-invariant action functional over $\mathcal{M}^{6}$. What is more, it implies automatic fulfilment of the classical version $E_{o}>s$ for the quantum massive condition $E_{o}>s+1$, for $s \geq 1$, which was postulated in Ref. [20] in the framework of a realization of the AdS group as a curved phase space. Namely, by construction, the theory is characterized by two parameters $\Delta_{1}$ and $\Delta_{2}$ which are the values of the second- and fourth-order Casimir phase-space functions, respectively, and can be algebraically reexpressed via some auxiliary parameters $E_{o}$ and $s$. Then it turns out that, first, the energy is positive definite over the phase space if and only if $E_{o} \geq s$; second, the energy possesses a global minimum when $E_{o}>s$. In the latter case, $E_{o}$ is the minimal value of the energy, while $s$ coincides with the total angular momentum at any phase-space point with the minimal energy. Therefore, the condition $E_{o}>s$ specifies massive spinning particles on the AdS space.

Another remarkable feature of the model is the fact that any physical observable, i.e. a gauge-invariant scalar field over the phase space, proves to be a function of the Hamilton generators of the AdS group only. As a result, the covariant quantization of the model is equivalent to realizing the unitary massive representations of the AdS group in function spaces over $\mathcal{M}^{6}$.

The paper is organized as follows. In section 2 we consider an AdS-covariant parametrization of $\mathcal{M}^{6}$ shown to be a homogeneous space of the AdS group. In section 3 the action functional of the model is derived, in an AdS-covariant way, and its local invariances are discussed both in the first- and second-order approaches. The main results of sections 2 and 3 can be easily extended to the cases of $d=3$ and higher dimensions, with $\mathcal{M}^{2(d-1)}=M^{d} \times S^{d-2}$ being the configuration space, where $M^{d}$ is a $d$-dimensional AdS space. In section 4 we investigate the conditions under which the energy is positive definite over the phase space and possesses a global positive minimum. Section 5 is devoted to the description of the model in terms of the inner $\mathcal{M}^{6}$-geometry. Here we also consider some obstructions to a straightforward generalization of our model to the case of arbitrary curved background. Dynamical histories with the minimal energy are studied in section 7 . In conclusion we discuss 
the results and some perspectives.

\section{Covariant realization for the configuration space}

We start with describing a covariant realization for the configuration space $\mathcal{M}^{6}=$ $M^{4} \times S^{2}$, where $M^{4}$ presents itself an ordinary anti-de Sitter space, $S^{2}$ a twodimensional sphere. It is useful for us to treat $M^{4}$ as a hyperboloid embedded into a five-dimensional pseudo-Euclidean space $\mathbf{R}^{3,2}$, with coordinates $y^{A}, A=5,0,1,2,3$, and defined by

$$
\eta_{A B} y^{A} y^{B}=-r^{2} \quad \eta_{A B}=\operatorname{diag}(--+++)
$$

$\mathcal{R}=-12 r^{-2}$ is the curvature of the AdS space. $M^{4}$ has the topology $S^{1} \times \mathbf{R}^{3}$ and, as a consequence, possesses closed timelike geodesics. That is why the universal covering space $\widetilde{M}^{4}$ of $M^{4}$ is standardly chosen to be the genuine AdS space. However, for studying the local physical properties, that we will be mainly interested in, one can equally well make use of $M^{4}$ as a model space. All our subsequent results are readily extended to the case of $\widetilde{M}^{4}$.

Similarly to $M^{4}, \mathcal{M}^{6}$ can be endowed with the structure of a homogeneous transformation space for an AdS group chosen below to be the connected component of the identity in $O(3,2)$ and denoted by $S O^{\uparrow}(3,2)$ (the elements of $S O^{\uparrow}(3,2)$ are specified by the conditions that their diagonal $2 \times 2$ and $3 \times 3$ submatrices, numbering by indices 5,0 and 1,2,3, respectively, have positive determinants). In order to explain this statement, let us consider the tangent bundle $T\left(M^{4}\right)$ that will be parametrized by 5 -vector variables $\left(y^{A}, b^{A}\right)$ under the constraints

$$
\begin{gathered}
y^{A} y_{A}=-r^{2} \\
y^{A} b_{A}=0 .
\end{gathered}
$$

The latter requirement simply expresses the fact that $b^{A} \partial / \partial y^{A}$ is a tangent vector to the point $y \in M^{4}$. Embedded into $T\left(M^{4}\right)$ is the $O(3,2)$-invariant subbundle $T_{l}\left(M^{4}\right)$ of non-zero lightlike tangent vectors

$$
\begin{aligned}
& b^{A} b_{A}=0 \\
& \left\{b^{A}\right\} \neq 0 .
\end{aligned}
$$

It turns out that $\mathcal{M}^{6}$ can be identified with the factor-space of $T_{l}\left(M^{4}\right)$ with respect to the equivalence relation

$$
b^{A} \sim \lambda b^{A} \quad \forall \lambda \in \mathbf{R} \backslash\{0\} .
$$

Really, there always exists a smooth mapping

$$
\mathcal{G}: M^{4} \rightarrow S O^{\uparrow}(3,2)
$$


such that $\mathcal{G}(y)$ moves a point $(y, b)$ at $T_{l}\left(M^{4}\right)$ to $(\mathbf{y}, \mathbf{b})$ having the form

$$
\mathbf{y}^{A}=\mathcal{G}^{A}{ }_{B}(y) y^{B}=(r, 0,0,0,0)
$$

and

$$
\begin{gathered}
\mathbf{b}^{A}=\mathcal{G}^{A}{ }_{B}(y) b^{B}=\left(0, \mathbf{b}^{a}\right) \quad a=0,1,2,3 \\
\eta_{a b} \mathbf{b}^{a} \mathbf{b}^{b}=0 \\
\left\{\mathbf{b}^{a}\right\} \neq 0 .
\end{gathered}
$$

For example, one can choose

$$
\mathcal{G}(y)=\left(\begin{array}{ccccc}
\frac{y^{5}}{r} & \frac{y^{0}}{r} & \vdots & -\frac{y^{1}}{r}-\frac{y^{2}}{r}-\frac{y^{3}}{r} \\
-\frac{y^{0}}{\rho} & \frac{y^{5}}{\rho} & 0 & 0 & 0 \\
\ldots \ldots \ldots & \ldots \ldots \ldots \ldots \ldots \ldots & \vdots & \ldots \ldots \ldots \\
-\frac{y^{1} y^{5}}{r \rho} & -\frac{y^{1} y^{0}}{r \rho} & & \\
-\frac{y^{2} y^{5}}{r \rho} & -\frac{y^{2} y^{0}}{r \rho} & \vdots & \delta^{i j}+\frac{y^{i} y^{j}}{r(\rho+r)} \\
-\frac{y^{3} y^{5}}{r \rho}-\frac{y^{3} y^{0}}{r \rho} & &
\end{array}\right)
$$

where

$$
\rho=\sqrt{\left(y^{5}\right)^{2}+\left(y^{0}\right)^{2}} \quad i, j=1,2,3 .
$$

From Eq. (7) we see that the fiber $\{(\mathbf{y}, \mathbf{b})\}$ over $\mathbf{y}$ in $T_{l}\left(M^{4}\right)$ looks exactly like the punctured light-cone in Minkowski space. The equivalence relation (4) proves to reduce the light-cone to $S^{2}$. Now, since the AdS group brings any equivalent points to equivalent ones, we conclude that $S O^{\uparrow}(3,2)$ naturally acts on the factor-space $M^{4} \times S^{2}$. Therefore, Eqs. (2-4) present an AdS-covariant realization of $\mathcal{M}^{6}$.

There exists some inherent arbitrariness in the choice of $\mathcal{G}$ defined by Eqs. (5) and (6). Such a mapping can be equally well replaced by another one

$$
\mathcal{G}^{\prime}{ }_{B}(y)=\Lambda_{C}^{A}(y) \mathcal{G}^{C}{ }_{B}(y)
$$

where $\Lambda$ takes it values in the stability group of the marked point $\mathbf{y}$

$$
\Lambda_{B}^{A}(y) \mathbf{y}^{B}=\mathbf{y}^{A}
$$

and has the general structure

$$
\begin{aligned}
\Lambda: M^{4} & \rightarrow S O^{\uparrow}(3,2) \\
\Lambda^{A}{ }_{B}(y) & =\left(\begin{array}{rrr}
1 & \vdots & 0 \\
\cdot & \vdots & \ldots \\
0 & \vdots & \Lambda_{b}^{a}(y)
\end{array}\right) \quad \Lambda_{b}^{a}(y) \in S O^{\uparrow}(3,1) .
\end{aligned}
$$


The set of all smooth mappings (10) forms an infinite-dimensional group isomorphic to a local Lorentz group of the AdS space. This group acts on $T\left(M^{4}\right)$ by the law

$$
(y, b) \longrightarrow\left(y, \mathcal{G}^{-1}(y) \Lambda(y) \mathcal{G}(y) b\right)
$$

$\mathcal{G}$ being a fixed solution of Eqs. (5), (6). As it is obvious, the local Lorentz group naturally acts on $\mathcal{M}^{6}$.

For writing down the explicit action of $S O^{\uparrow}(3,2)$ on $\mathcal{M}^{6}$, it appears useful from the very beginning to replace the AdS-covariant parametrization $\left(y^{A}, b^{A}\right)$ of $T\left(M^{4}\right)$ with a Lorentz-covariant one $\left(y^{A}, \mathbf{b}^{a}\right)$, where the 4 -vector $\mathbf{b}^{a}$ is related to $b^{A}$ as in Eq. (7.a). Given a group element $g \in S O^{\uparrow}(3,2)$, it moves $(y, b)$ to $(g y, g b)$, hence $(y, \mathbf{b})$ to $\left(g y, \Lambda_{g}(y) \mathbf{b}\right)$, where

$$
\Lambda_{g}(y) \equiv \mathcal{G}(g y) g \mathcal{G}^{-1}(y)
$$

is a Lorentz transformation of the form (10). One readily finds

$$
\Lambda_{g_{1}}(y) \Lambda_{g_{2}}(y)=\Lambda_{g_{1} g_{2}}(y)
$$

for arbitrary $g_{1}, g_{2} \in S O^{\uparrow}(3,2)$. We thus arrive at a nonlinear representation of the AdS group. Now, the problem simly reduces to making use of the known action of the Lorentz group on the light-cone $(7 \cdot \mathrm{a}-\mathrm{c})$.

The covariant realization of $\mathcal{M}^{6}$ described is based on the use of a lightlike vector variable to parametrize $S^{2}$. Another realization, which involves a constrained spinor variable and appears to be most suited for constructing generalized AdS-coherent states, will be given in a forthcoming publication [21].

\section{Derivation of the action functional}

We set about deriving the action functional of a point particle on $\mathcal{M}^{6}$. Our main dynamical principle is the requirement of strong conservation for classical counterparts of the Casimir operators of $s o(3,2)$. Let us recall that these operators can be chosen (see, for example, [11]) in the form

$$
\begin{gathered}
\hat{\Omega}_{1}=\frac{1}{2} \hat{L}^{A B} \hat{L}_{A B} \\
\hat{\Omega}_{2}=\hat{W}^{A} \hat{W}_{A}
\end{gathered}
$$

where $\hat{W}^{A}$ is the Pauli-Lubanski vector

$$
\hat{W}^{A}=\frac{1}{8} \varepsilon^{A B C D E} \hat{L}_{B C} \hat{L}_{D E}
$$

$\hat{L}_{A B}$ being the generators of $s o(3,2)$. 
We start with an extended phase space parametrized by unconstrained coordinates $y^{A}, b^{A}$ and their canonical momenta $p_{A}, k_{A}$ with respect to the standard Poisson structure

$$
\left\{y^{A}, p_{B}\right\}=\left\{b^{A}, k_{B}\right\}=\delta_{B}^{A}
$$

other brackets vanish. Obviously, the AdS group acts on the phase space by canonical transformations. This action induces special representation of this group in the space of smooth functions on the phase space. For an infinitesimal group transformation

$$
\delta y^{A}=\theta_{B}^{A} y^{B} \quad \theta_{A B}=-\theta_{B A}
$$

the corresponding change of a phase-space function $\Phi(y, b, p, k)$ is given in terms of the Poisson bracket as follows

$$
\delta \Phi=\frac{1}{2} \theta^{A B}\left\{\mathcal{J}_{A B}, \Phi\right\}
$$

where the Hamilton generators read

$$
\mathcal{J}_{A B}=p_{A} y_{B}+k_{A} b_{B}-(A \leftrightarrow B) .
$$

Next we introduce two sets of AdS invariant phase-space constraints: kinematical

$$
\begin{gathered}
T_{1}=y^{2}+r^{2} \\
T_{2}=(y, p) \\
T_{3}=(y, b) \\
T_{4}=(y, k) \\
T_{5}=b^{2} \\
T_{6}=(b, k)
\end{gathered}
$$

and dynamical

$$
\begin{gathered}
-T_{7}=\Omega_{1}-\Delta_{1} \\
T_{8}=\Omega_{2}-\Delta_{2} .
\end{gathered}
$$

Here $\Delta_{1}$ and $\Delta_{2}$ are constant parameters. Evolution of the mechanical system is postulated to develop on the full surface of constraints

$$
T_{I} \approx 0 \quad I=1, \ldots, 8 .
$$

Let us shortly discuss the structure and physical origion of the constraints. The kinematical constraints are introduced in order to define $6+6$ dimensional phase space over $\mathcal{M}^{6}$, while the dynamical ones lead to unique choice of the action functional. The constraints $T_{1}, \ldots, T_{4}$ determine the cotangent bundle of $T\left(M^{4}\right)$, and $T_{6}$ generates 
the equivalence relation (4). With respect to the Poisson bracket (16), the full set of constraints is decomposed into two groups: second-class constraints $T_{1}, \ldots, T_{4}$

$$
\frac{1}{2}\left\{T_{1}, T_{2}\right\}=\left\{T_{3}, T_{4}\right\}=-r^{2}
$$

and first-class constraints $T_{5}, \ldots, T_{8}$. The constraints $T_{1}, \ldots, T_{4}$ can be eliminated by means of introducing local independent coordinates on $M^{4}$. To keep the AdScovariance explicitly, however, we prefer to use the constrained variables. It is worth noting that the constraints $T_{2}$ and $T_{4}$ can be treated as a particular gauge fixing for the set of first-class constraints $T_{1}, T_{3}, T_{5}, \ldots, T_{8}$. Our subsequent results could be obtained without imposing $T_{2}$ and $T_{4}$, but the use of these constraints leads to maximally simple expressions for the classical counterparts of the operators (14)

$$
\Omega_{1} \approx-r^{2} p^{2} \quad \Omega_{2} \approx r^{2}(b, p)^{2} k^{2} .
$$

Assuming the theory to be reparametrization invariant, the Hamiltonian is a linear combination of the constraints and the first-order (Hamilton) action reads

$$
S=\int \mathrm{d} \tau\left(p_{A} \dot{y}^{A}+k_{A} \dot{b}^{A}-\frac{1}{2} \sum_{I=1}^{8} \lambda_{I} T_{I}\right) .
$$

Here $\tau$ is the evolution parameter, $\lambda$ 's are Lagrange multipliers associated to the constraints. The action can be readily brought to a second-order (Lagrange) form by eliminating the momenta $p_{A}, k_{A}$ and kinematical multipliers $\lambda_{1}, \ldots, \lambda_{6}$ with the aid of their equation of motion

$$
\frac{\delta S}{\delta p_{A}}=\frac{\delta S}{\delta k_{A}}=\frac{\delta S}{\delta \lambda_{i}}=0 \quad i=1, \ldots, 6 .
$$

This leads to

$$
\begin{gathered}
S=\int \mathrm{d} \tau \mathcal{L} \\
\mathcal{L}=\frac{1}{2 e_{1}}\left(\dot{y}^{2}-\frac{\Delta_{1}}{r^{2}} e_{1}^{2}\right)+\frac{1}{2 e_{2}}\left(\left(\frac{\dot{b}^{2}}{(\dot{y}, b)^{2}}+\frac{1}{r^{2}}\right) e_{1}^{2}+\frac{\Delta_{2}}{r^{2}} e_{2}^{2}\right) .
\end{gathered}
$$

Here $e_{1} \equiv r^{2} \lambda_{7}, e_{2} \equiv r^{2} \lambda_{8}$ and the variables $y^{A}$ and $b^{A}$ are restricted to satisfy the holonomic constraints (18.a), (19.a) and (20).

The first-order action possesses four gauge invariances associated with the firstclass constraints $T_{5}, \ldots, T_{8}$. After passing to the second-order action, there remain only three gauge symmetries related to $T_{6}, T_{7}$ and $T_{8}$. Each gauge transformation can be represented as a combination of some reparametrization of the world line

$$
\delta_{\epsilon} y^{A}=\epsilon \dot{y}^{A}, \quad \delta_{\epsilon} b^{A}=\epsilon \dot{b}^{A}, \quad \delta_{\epsilon} e_{i}=\frac{d}{d \tau}\left(\epsilon e_{i}\right) \quad i=1,2
$$

local $b$-rescaling

$$
\delta_{\mu} b^{A}=\mu b^{A}, \quad \delta_{\mu} y^{A}=\delta_{\mu} e_{i}=0
$$


and reparametrization-like transformation of the form

$$
\delta_{\nu} y^{A}=\nu p^{A}, \quad \delta_{\nu} b^{A}=\frac{\nu}{r^{2}} \frac{(\dot{y}, b)}{e_{1}} y^{A}, \quad \delta_{\nu} e_{1}=\dot{\nu}, \quad \delta_{\nu} e_{2}=0
$$

where

$$
p^{A}=\frac{\dot{y}^{A}}{e_{1}}-\frac{e_{1}^{2}}{e_{2}(\dot{y}, b)^{3}}\left\{\dot{b}^{2}+\frac{1}{r^{2}}(\dot{y}, b)^{2}\right\} b^{A} .
$$

Here $\epsilon, \nu$ and $\mu$ are arbitrary, modulo boundary conditions, functions of $\tau$.

It should be pointed out that all gauge symmetries (30-32) of the action (29) remain intact in the case when $y^{A}$ and $b^{A}$ are considered to be $d+1$ vectors in $\mathbf{R}^{d-1,2}$ subject to Eqs. $(2,3)$. Therefore, we naturally obtain a model of a spinning particle in $d$-dimensional AdS space $\mathcal{M}^{d}$. The gauge invariance (31) implies that the configuration space of the model is $\mathcal{M}^{d} \times S^{d-2}$.

\section{Physical observables, energy and spin}

The Hamilton generators (17) determine the transformation law of phase-space functions under the action of the AdS group. On the other hand, they generate the total set of physical observables of the system. A phase-space function $\mathcal{F}$ is said to be a (strong) physical observable if it commutes with the first-class constraints

$$
\left\{\mathcal{F}, T_{I}\right\}_{D B}=0 \quad I=5, \ldots, 8
$$

with respect to the Dirac bracket related to the second-class constraints $T_{1}, \ldots, T_{4}$ :

$$
\begin{gathered}
\left\{y_{A}, y_{B}\right\}_{D B}=\left\{y_{A}, b_{B}\right\}_{D B}=\left\{y_{A}, k_{B}\right\}_{D B}=\left\{b_{A}, b_{B}\right\}_{D B}=\left\{k_{A}, k_{B}\right\}_{D B}=0 \\
\left\{p_{A}, p_{B}\right\}_{D B}=-\frac{1}{r^{2}} \mathcal{J}_{A B} \\
\left\{y_{A}, p_{B}\right\}_{D B}=\left\{b_{A}, k_{B}\right\}_{D B}=\eta_{A B}+\frac{1}{r^{2}} y_{A} y_{B} \\
\left\{b^{A}, p^{B}\right\}_{D B}=\frac{1}{r^{2}} y_{A} b_{B} \quad\left\{k^{A}, p^{B}\right\}_{D B}=\frac{1}{r^{2}} y_{A} k_{B} .
\end{gathered}
$$

By direct analysis one can show that any physical observable is a function of the Hamilton generators only

$$
\mathcal{F}=\mathcal{F}\left(\mathcal{J}_{A B}\right)
$$

on the total surface of constraints. The same fact follows from more simple consideration. Because of the presence of four second-class and four first-class constraints, physical phase space is eight-dimensional. Hence it can be parametrized by 10 variables $\mathcal{J}_{A B}$ subject to the constraints $T_{7}, T_{8}$. As a result, the Hamilton generators of the AdS group completely specify gauge-invariant properties of the system. 
We turn now to more deep study of the constraints $(22,23)$. It will be shown that for special values of $\Delta_{1}$ and $\Delta_{2}$ the system is characterized by two physical parameters: minimal positive energy $E_{o}$ and spin $s$, energy $E$ of the particle being defined as follows

$$
E \equiv \mathcal{J}^{05}=p^{0} y^{5}-p^{5} y^{0}+k^{0} b^{5}-k^{5} b^{0} .
$$

Below we will assume $\Delta_{1}$ and $\Delta_{2}$ to be positive, what implies $p^{A}$ is a timelike 5 -vector and $k^{A}$ is a spacelike one.

The fact that the energy (37) might be globally positive definite, on a connected component of the constrained surface, lies mainly in the algebraic structure of the AdS group. For recalling let us consider, for a moment, the model of a massive spinless particle on the AdS space with the Lagrangian

$$
\mathcal{L}=\frac{1}{2 e}\left(\dot{y}^{2}-\frac{\Delta}{r^{2}} e^{2}\right) \quad \Delta>0
$$

which is dynamically equivalent to our model in the case $\Delta_{2}=0$. Here the constrained surface is given by

$$
\mathcal{T}_{1}=y^{2}+r^{2} \quad \mathcal{T}_{2}=(y, p) \quad \mathcal{T}_{3}=r^{2} p^{2}+\Delta .
$$

and, as a topological space, consists of two connected components determined by the AdS-covariant conditions

$$
\begin{aligned}
& p^{0} y^{5}-p^{5} y^{0}>0 \\
& p^{5} y^{0}-p^{0} y^{5}<0 .
\end{aligned}
$$

Really, having mutually orthogonal timelike 5-vectors $p=\left(p^{A}\right)$ and $y=\left(y^{A}\right)$ one can construct an orthonormal 5-frame $\left\{h_{B}\right\}$

$$
h_{B}=\left(h_{B}^{A}\right) \quad\left(h_{B}, h_{C}\right)=\eta_{B C}
$$

such that $h_{5}=\frac{1}{r} y, h_{0}=\frac{r}{\sqrt{\Delta}} p$. The matrix

$$
h=\left(h_{B}^{A}\right)
$$

belongs to $O(3,2)$, hence

$$
\left|h^{5}{ }_{5} h^{0}{ }_{0}-h^{5}{ }_{0} h^{0}{ }_{5}\right| \geq 1 .
$$

It is now obvious that sign of the energy

$$
\mathcal{E}=p^{0} y^{5}-p^{5} y^{0}
$$

is AdS-invariant as well as we have

$$
|\mathcal{E}| \geq \sqrt{\Delta}
$$


at any point of the constrained surface. Direct computation of $\mathcal{E}$ for the phase-space domain (40.a) leads to the expression

$$
\mathcal{E}=\sqrt{\Delta\left(\frac{\rho}{r}\right)^{2}+\rho^{2} \vec{p}^{2}-(\vec{y}, \vec{p})^{2}} .
$$

Similarly to the spinless case, the constrained surface in our model involves two connected components specified by the conditions (40). Let us analyse the function of energy (37) for the points of the component (40.a). It is useful to express the variables $(p, b, k)$, parametrizing the fiber over some point $y$ of the AdS space, via those for the fiber over the marked point (6)

$$
(p, b, k)=\mathcal{G}^{-1}(y)(\mathbf{p}, \mathbf{b}, \mathbf{k})
$$

$\mathcal{G}(y)$ is given by Eq. (8). We have

$$
\begin{aligned}
& \mathbf{p}^{A}=\left(0, \mathbf{p}^{0}, \overrightarrow{\mathbf{p}}\right) \quad \mathbf{p}^{0}=\sqrt{\frac{\Delta_{1}}{r^{2}}+\overrightarrow{\mathbf{p}}^{2}} \\
& \mathbf{b}^{A}=\left(0, \mathbf{b}^{0}, \overrightarrow{\mathbf{b}}\right) \quad\left|\mathbf{b}^{0}\right|=|\overrightarrow{\mathbf{b}}| \neq 0 \\
& \mathbf{k}^{A}=\left(0, \mathbf{k}^{0}, \overrightarrow{\mathbf{k}}\right) \quad \mathbf{k}^{0}=\frac{1}{\mathbf{b}^{0}}(\overrightarrow{\mathbf{b}}, \overrightarrow{\mathbf{k}})
\end{aligned}
$$

where we have accounted the constraints. The momenta $p$ and $\mathbf{p}$ are related by the rule

$$
\begin{aligned}
p^{5} & =-\frac{y^{0}}{\rho} \mathbf{p}^{0}+\frac{y^{5}}{r \rho}(\vec{y}, \overrightarrow{\mathbf{p}}) \\
p^{0} & =\frac{y^{5}}{\rho} \mathbf{p}^{0}+\frac{y^{0}}{r \rho}(\vec{y}, \overrightarrow{\mathbf{p}}) \\
\vec{p} & =\overrightarrow{\mathbf{p}}+\frac{\vec{y}}{r(\rho+r)}(\vec{y}, \overrightarrow{\mathbf{p}})
\end{aligned}
$$

and similarly for the rest variables. Now, one readily finds

$$
E=\rho \mathbf{p}^{0}-\frac{1}{r} \mathbf{b}^{0}(\vec{y}, \vec{w})
$$

where

$$
\vec{w}=\overrightarrow{\mathbf{k}}-\overrightarrow{\mathbf{b}} \frac{(\overrightarrow{\mathbf{b}}, \overrightarrow{\mathbf{k}})}{\overrightarrow{\mathbf{b}}^{2}} .
$$

It is important that the 3 -vector just introduced possesses the properties

$$
(\vec{w}, \overrightarrow{\mathbf{b}})=0 \quad \vec{w}^{2}=\mathbf{k}^{2}=k^{2} .
$$

Then, further use of the constraints allows to get the following unequality

$$
\left|\frac{1}{r} \mathbf{b}^{0}(\vec{y}, \vec{w})\right| \leq \frac{\sqrt{\Delta_{2}}}{\Delta_{1}}|\vec{y}|\left(\mathbf{p}^{0}+|\overrightarrow{\mathbf{p}}|\right)
$$


and hence

$$
E \geq \rho \mathbf{p}^{0}-\frac{\sqrt{\Delta_{2}}}{\Delta_{1}}|\vec{y}|\left(\mathbf{p}^{0}+|\overrightarrow{\mathbf{p}}|\right) .
$$

The expressions in both sides coincide under the conditions

$$
\vec{y}\|\vec{w} \quad \overrightarrow{\mathbf{p}}\| \overrightarrow{\mathbf{b}} \quad \mathbf{b}^{0}>0
$$

and therefore

$$
\vec{y} \perp \vec{b} \quad \vec{p} \| \vec{b}
$$

as a consequence of Eqs. (47), (49.b). Here we have used the fact that the energy is invariant under the transformations $\mathbf{b} \rightarrow \lambda \mathbf{b}, \mathbf{k} \rightarrow \lambda^{-1} \mathbf{k}$, hence $\mathbf{b}^{0}$ can be chosen to be positive.

Because of Eq. (51), the lower boundary of values of the energy $E=E(y, p, b, k)$ is given by the function

$$
\begin{gathered}
f(u, v)=\sqrt{\left(r^{2}+u^{2}\right)\left(\frac{\Delta_{1}}{r^{2}}+v^{2}\right)}-\frac{\sqrt{\Delta_{2}}}{\Delta_{1}} u\left(v+\sqrt{\frac{\Delta_{1}}{r^{2}}+v^{2}}\right) \\
u=|\vec{y}| \quad v=|\vec{p}| \quad 0 \leq u, v \leq \infty .
\end{gathered}
$$

This function proves to be positive definite if and only if

$$
2 \sqrt{\Delta_{2}} \leq \Delta_{1}
$$

Thus the case $\Delta_{1}<2 \sqrt{\Delta_{2}}$ is unphysical, for the energy can take zero and negative values, and should be ruled out from our consideration.

The choice

$$
2 \sqrt{\Delta_{2}}=\Delta_{1}
$$

is very special, since the energy can sink down to zero in a limit of infinite $|\vec{y}|$ and $|\vec{p}|$. A similar situation takes place for the massless spinless particle (see Eq. (44) with $\Delta=0$ ) ; here the energy is positive definite but can approach zero for $|\vec{p}| \rightarrow 0$. Finally, the case

$$
2 \sqrt{\Delta_{2}}<\Delta_{1}
$$

is characterized by the property

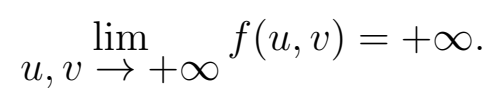

The same behaviour is characteristic of the massive spinless particle, in accordance with Eq. (44). Thus we are tempted to treat the choices (55) and (56) to be characteristic of massless and massive spinning particles, respectively. From now on, we restrict ourselves to the consideration of the case (56). 
Introducing new parameters $E_{o}$ and $s$ defined by

$$
E_{o}^{2} \equiv \frac{\Delta_{1}}{2}+\sqrt{\left(\frac{\Delta_{1}}{2}\right)^{2}-\Delta_{2}} \quad s^{2} \equiv \frac{\Delta_{1}}{2}-\sqrt{\left(\frac{\Delta_{1}}{2}\right)^{2}-\Delta_{2}}
$$

Eq. (56) is seen to be equivalent to the relation

$$
s<E_{o}
$$

and the original parameters are expressed as follows

$$
\begin{gathered}
\Delta_{1}=E_{o}^{2}+s^{2} \\
\Delta_{2}=E_{o}^{2} s^{2} .
\end{gathered}
$$

In the domain of non-zero $u$ and $v, f$ proves to possess the only extremal point

$$
\begin{gathered}
|\vec{y}|=\frac{r s}{\sqrt{E_{o}^{2}-s^{2}}} \\
|\vec{p}|=\frac{s^{2}}{r \sqrt{E_{o}^{2}-s^{2}}}
\end{gathered}
$$

in which $f$ is equal to $E_{o}$. Then, Eq. (57) and the obvious relations

$$
E_{o}<f(0, v) \quad E_{o}<f(u, 0)
$$

imply that $E_{o}$ is the global minimum of the energy

$$
E \geq E_{o}
$$

The global minimum is achieved in those points of the constrained surface which obey the relations (52) and (61). In every such point, the Hamilton generators turn out to have the form

$$
\mathcal{J}^{A B}=\left(\begin{array}{cccc}
0 & -E_{o} & \vdots & 0 \\
E_{o} & 0 & \vdots & 0 \\
\cdots & \ldots & \vdots & \ldots \\
0 & 0 & \vdots & \mathcal{J}^{i j}
\end{array}\right) \quad \sum_{i<j}\left(\mathcal{J}^{i j}\right)^{2}=s^{2}
$$

and the classical Pauli-Lubanski vector (15) reads

$$
W^{A}=\left(0,0, W^{i}\right) \quad W^{i}=\frac{1}{2} E_{o} \varepsilon^{i j k} \mathcal{J}^{j k} .
$$

Thus, the parameter $s$ has the sence of the total angular momentum of a particle with the minimal energy. That is why we can identify $s$ with spin. 
It should be remarked that the massive spinning particle having the minimal energy remains to stay at the finite distance (61.a) from the origion $\vec{y}=0$ and its "3-momentum" has the constant non-zero value (61.b). The particle moves along

a circle, with center at the origion, that lies in the plane orthogonal to $\vec{W}$ (64.b). This picture drastically differs from that for the massive spinless particle which gets the minimum of the energy only when $\vec{y}=0$ and $\vec{p}=0$. In accordance with Eq. (61), the dynamical behaviour of the spinning particle looks similar to that of the spinless one only when $E_{o} \gg s$. For $E_{o} \approx s$, however, the spin effects become very strong and cause the particle with $E=E_{o}$ to be located far away from the origion. Another important remark is that the conditions (61), characterizing the states with the minimal energy, are invariant with respect to the gauge transformations induced by the constraints.

\section{$5 \quad$ Spinning particle and inner AdS-geometry}

In this section, the model will be reformulated as a dynamical system on a curved space. We start with resolving the second-class constraints $(18,19)$ and the Dirac bracket (35) via unconstrained variables on the cotangent bundle of $T\left(M^{4}\right)$ and related geometric objects. Then we describe the model in terms of inner coordinates on $\mathcal{M}^{6}$.

Let $x^{m}, m=0,1,2,3$, be local coordinates on the surface (1). The induced metric $\mathrm{d} s^{2}=\eta_{A B} \mathrm{~d} y^{A} \mathrm{~d} y^{B}$ reads

$$
\mathrm{d} s^{2}=g_{m n}(x) \mathrm{d} x^{m} \mathrm{~d} x^{n}
$$

$g_{m n}$ being a metric of constrant negative curvature $\mathcal{R}=-12 / r^{2}$. Now the cotangent bundle of $T\left(M^{4}\right)$ can be parametrized by unconstrained variables $\left(x^{m}, \mathbf{p}^{a}, \mathbf{b}^{a}, \mathbf{k}^{a}\right)$, where $\mathbf{p}, \mathbf{b}$ and $\mathbf{k}$ are defined by the rule $(45)$, for $\mathcal{G}(y)$ a solution of Eqs. $(5,6)$. Obviously, $\mathbf{p}, \mathbf{b}$ and $\mathbf{k}$ transform as 4 -vectors with respect to the local Lorentz group and as scalars under the general covariance group of $M^{4}$. Associated with $\mathcal{G}(y)$ is a vierbein $e_{m}{ }^{a}(x)$ of the metric that converts curved-space indices into flat-space ones. Really, let us define

$$
e_{m}{ }^{A} \equiv \mathcal{G}^{A}{ }_{B} \frac{\partial y^{B}}{\partial x^{m}}=-\frac{\partial \mathcal{G}^{A}{ }_{B}}{\partial x^{m}} y^{B}=\left(0, e_{m}{ }^{a}\right)
$$

where we have used the identity

$$
\mathcal{G}^{5}{ }_{B}=-\frac{1}{r} y_{B}
$$

Since $\mathcal{G}(y)$ belongs to $O(3,2)$, one readily gets the relations

$$
g_{m n}=e_{m}{ }^{a} e_{n}{ }^{b} \eta_{a b}
$$


By construction, the functions $x(y)$ and $\mathcal{G}(y)$ are defined only on the AdS hyperboloid. They can be uniquely extended onto the subspace of $\mathbf{R}^{3,2}$ [13]

$$
\mathcal{W}=\left\{y \in \mathbf{R}^{3,2}, \quad y^{2}<0\right\}
$$

if one restricts them to have zeroth order of homogeneity in $y$

$$
\frac{\partial x^{m}}{\partial y^{C}} y^{C}=0 \quad \frac{\partial \mathcal{G}^{A} B}{\partial y^{C}} y^{C}=0 .
$$

Thus the variables $x^{m}$ and $\sigma, \sigma \equiv\left(-y^{A} y_{A}\right)^{-1 / 2}$, can be chosen to parametrize $\mathcal{W}$ instead of $y^{A}$ (note, $\sigma$ commutes with any phase-space function with respect to the Dirac bracket (35)). Introducing

$$
e_{A}^{m} \equiv \frac{\partial x^{m}}{\partial y^{B}}\left(\mathcal{G}^{-1}\right)^{B}{ }_{A}=\left(0, e_{a}{ }^{m}\right)
$$

one finds

$$
e_{a}^{m} e_{m}^{b}=\delta_{a}^{b}
$$

therefore $e_{a}^{m}(x)$ is the inverse vierbein.

Another geometric object, the torsion-free spin connection $\omega_{m a b}(x)$, defined by

$$
\omega_{m a b}=-\omega_{m b a} \quad T_{m n}{ }^{a}=\partial_{n} e_{m}{ }^{a}-\partial_{m} e_{n}{ }^{a}+\omega_{n}{ }^{a}{ }_{b} e_{m}{ }^{b}-\omega_{m}{ }^{a}{ }_{b} e_{n}{ }^{b}=0
$$

can be represented in the form

$$
\omega_{m}{ }^{a}{ }_{b}=\mathcal{G}^{a}{ }_{C} \frac{\partial\left(\mathcal{G}^{-1}\right)^{C}}{\partial x^{m}} .
$$

Let us pass from $\mathbf{p}_{a}$ to new variables $p_{m}$ defined as follows

$$
\mathbf{p}_{a}=e_{a}{ }^{m} \mathbf{p}_{m} \quad \mathbf{p}_{m}=p_{m}-\frac{1}{2} \omega_{m c d} \mathcal{M}^{c d}
$$

where

$$
\mathcal{M}^{c d}=\mathbf{k}^{c} \mathbf{b}^{d}-\mathbf{k}^{d} \mathbf{b}^{c} .
$$

It is now an instructive exercise to verify, with the help of Eqs. (66-73), that the Dirac brackets (35) are equivalent to the canonical commutation relations for the variables $x^{m}, p_{n}, \mathbf{b}^{a}$ and $\mathbf{k}_{b}$ :

$$
\left\{x^{m}, p_{n}\right\}_{D B}=\delta_{n}^{m} \quad\left\{\mathbf{b}^{a}, \mathbf{k}_{b}\right\}_{D B}=\delta_{b}^{a}
$$

the other brackets vanish. Eq. (75) defines the Hamilton generators of the Lorentz transformations acting on $\mathbf{b}^{a}$ and $\mathbf{k}_{b}$ and leaving $x^{m}$ and $p_{n}$ inert. In the phase-space variables inroduced, the second class constraints are completely eliminated and we 
rest with the first-class constraints (20-21). Finally, the Lagrangian (29) takes the form

$$
\mathcal{L}=\frac{1}{2 e_{1}}\left(g_{m n} \dot{x}^{m} \dot{x}^{n}-\frac{\Delta_{1}}{r^{2}} e_{1}^{2}\right)+\frac{1}{2 e_{2}}\left(\frac{\dot{\mathbf{b}}^{a} \dot{\mathbf{b}}_{a}}{\left(\dot{x}^{m} e_{m}{ }^{c} \mathbf{b}_{c}\right)^{2}} e_{1}^{2}+\frac{\Delta_{2}}{r^{2}} e_{2}^{2}\right)
$$

where

$$
\dot{\mathbf{b}}^{a}=\dot{\mathbf{b}}^{a}+\dot{x}^{m} \omega_{m}{ }^{a}{ }_{c} \mathbf{b}^{c}
$$

is the Lorentz-covariant derivative of $\mathbf{b}^{a}$ along the world-line. Here $\mathbf{b}^{a}$ is constrained to be a lightlike 4 -vector.

The above Lagrangian is a minimal curved-space extension of that recently proposed in Ref. [6] for the massive spinning particle in Minkowski space:

$$
\mathcal{L}_{\text {flat }}=\frac{1}{2 e_{1}}\left(\dot{x}^{a} \dot{x}_{a}-\left(m e_{1}^{2}\right)\right)+\frac{1}{2 e_{2}}\left(\frac{\dot{\mathbf{b}}^{a} \dot{\mathbf{b}}_{a}}{\left(\dot{x}^{c} \mathbf{b}_{c}\right)^{2}} e_{1}^{2}+\left(m s e_{2}\right)^{2}\right) .
$$

The action functional in Minkowski space possesses three types of local symmetries which can be read off from Eqs. (30-33) by making obvious replacements and setting $r^{-2}=0$. The Lagrangian (77) is formally well-defined not only for the AdS space but for arbitrary curved space-time. However, choosing in (77) $e_{m}{ }^{a}(x)$ to be the vierbein of a space-time with non-constant curvature $\mathcal{R}_{m n a b}$, the action turns out to be invariant only under the general coordinate transformations and local $b$-rescalings. The existence of three local symmetries takes place if and only if the curvature of space-time is constant. This can be seen as follows. The "covariant derivatives" (74) satisfy the commutation relations

$$
\left\{\mathbf{p}_{m}, \mathbf{p}_{n}\right\}=-\frac{1}{r^{2}} e_{m}{ }^{a} e_{n}{ }^{b} \mathcal{M}_{a b}
$$

in the AdS space and

$$
\left\{\mathbf{p}_{m}, \mathbf{p}_{n}\right\}=\frac{1}{2} \mathcal{R}_{m n}{ }^{a b} \mathcal{M}_{a b}
$$

in general case. For the constraints $T_{7}$ and $T_{8}(22,23,26)$ rewritten in the form

$$
\begin{gathered}
\frac{1}{r^{2}} T_{7}=g^{m n} \mathbf{p}_{m} \mathbf{p}_{n}+\frac{\Delta_{1}}{r^{2}} \approx 0 \\
\frac{1}{r^{2}} T_{8}=\left(\mathbf{b}^{a} e_{a}{ }^{m} \mathbf{p}_{m}\right)^{2} \mathbf{k}^{c} \mathbf{k}_{c}-\frac{\Delta_{2}}{r^{2}} \approx 0
\end{gathered}
$$

Eqs. (76), (81) give

$$
\frac{1}{r^{4}}\left\{T_{7}, T_{8}\right\}=2(\mathbf{p}, \mathbf{b}) \mathbf{k}^{2} \mathbf{b}^{a} \mathbf{p}^{b} \mathcal{R}_{a b c d} \mathcal{M}^{c d}
$$

The expression in the r.h.s of (83) vanishes on the surface of constraints $T_{5}, T_{6}$ if and only if

$$
\mathcal{R}_{a b c d}=\frac{1}{12} \mathcal{R}\left(\eta_{a c} \eta_{b d}-\eta_{a d} \eta_{b c}\right)
$$


where the scalar curvature $\mathcal{R}$ is a constant, as a consequence of the Bianchi identities. Therefore, the constraints $T_{5}, \ldots, T_{8}$ can form a first-class algebra only in the case of space-times with constant curvature.

Now, let us reformulate the model in terms of internal variables on $\mathcal{M}^{6}$. We standardly cover $S^{2}=\mathbf{C} \cup\{\infty\}$, considered as a one-dimensional complex manifold, by two open patches $U_{1}=\mathbf{C}$ and $U_{2}=\mathbf{C}^{*} \cup\{\infty\}$ parametrized by complex coordinates $z$ and $\omega$, respectively, which are related in the overlap $U_{1} \cap U_{2}=\mathbf{C}^{*}$ by the rule $\omega=-1 / z$.

The Lorentz group $S O^{\uparrow}(3,1) \cong S L(2, \mathbf{C}) / \mathbf{Z}_{2}$ acts on $S^{2}$ by fractional linear transformations of the form

$$
z^{\prime}=\frac{a z-b}{-c z+d} \quad\|N\|=N_{\alpha}^{\beta}=\left(\begin{array}{ll}
a & b \\
c & d
\end{array}\right) \in S L(2, \mathbf{C}) .
$$

This implies that the two-component objects

$$
\begin{gathered}
z^{\alpha}=(z)^{\alpha}=(1, z) \quad \alpha=0,1 \\
\omega_{\alpha}=(\omega)^{\alpha}=(1, \omega)=-z^{-1} \varepsilon_{\alpha \beta} z^{\beta}
\end{gathered}
$$

transform under the action of the Lorentz group (84) simultaneously as left Weyl spinors and spinor fields on $S^{2}$ :

$$
\begin{aligned}
z^{\prime \alpha} & =\left(\frac{\partial z^{\prime}}{\partial z}\right)^{1 / 2} z^{\beta}\left(N^{-1}\right)_{\beta}{ }^{\alpha} \\
\omega_{\alpha}^{\prime} & =\left(\frac{\partial \omega^{\prime}}{\partial \omega}\right)^{1 / 2}(N)_{\alpha}{ }^{\beta} \omega_{\beta} .
\end{aligned}
$$

Our two-component spinor notations and conventions coincide with those adopted in [22], in particular, $\varepsilon_{\alpha \beta}$ is the spinor metric.

The lightlike variable $\mathbf{b}^{a}$, entering the Lagrangian (77), can be parametrized as follows

$$
\mathbf{b}^{a}=\xi^{a} \varphi(\mathbf{b})=\zeta^{a} \psi(\mathbf{b})
$$

where

$$
\begin{aligned}
\xi^{a} & =\left(\sigma^{a}\right)_{\alpha \dot{\alpha}} z^{\alpha} \bar{z}^{\dot{\alpha}} \\
\zeta^{a} & =\left(\sigma^{a}\right)_{\alpha \dot{\alpha}} \omega^{\alpha} \bar{\omega}^{\dot{\alpha}}
\end{aligned}
$$

and

$$
\psi(\mathbf{b})=|\omega|^{2} \varphi(\mathbf{b})
$$

for some function $\varphi(\mathbf{b})$. Here $\left(\sigma^{a}\right)_{\alpha \dot{\alpha}}$ are the Pauli matrices [22], $\bar{z}^{\dot{\alpha}}$ and $\bar{\omega}^{\dot{\alpha}}$ are the complex conjugates of $z^{\alpha}$ and $\omega^{\alpha}$, respectively. Then, with the use of the identity

$$
\varepsilon^{\alpha \beta}=z^{\alpha} \partial_{z} z^{\beta}-z^{\beta} \partial_{z} z^{\alpha}
$$


the Lagrangian (77) can be rewritten in the form

$$
\mathcal{L}=\frac{1}{2 e_{1}}\left(g_{m n} \dot{x}^{m} \dot{x}^{n}-\frac{\Delta_{1}}{r^{2}} e_{1}^{2}\right)+\frac{1}{2 e_{2}}\left(\frac{4|\dot{z}|^{2}}{\left(\dot{x}^{m} e_{m}{ }^{a} \xi_{a}\right)^{2}} e_{1}^{2}+\frac{\Delta_{2}}{r^{2}} e_{2}^{2}\right)
$$

where

$$
\begin{gathered}
\dot{z}=\dot{z}+\frac{1}{2} \dot{x}^{m} \omega_{m a b}(x) \Sigma^{a b} \\
\Sigma^{a b}=-\left(\sigma^{a b}\right)_{\alpha \beta} z^{\alpha} z^{\beta}
\end{gathered}
$$

with $\sigma^{a b}$ the spin matrices [22]. Contrary to $\dot{z}, \dot{z}$ is changed homogeneously with respect to the local Lorentz transformations.

The multipliers $e_{1}$ and $e_{2}$ can be eliminated with the aid of their equations of motion. Then $\mathcal{L}$ turns into

$$
\mathcal{L}=-\frac{1}{r} \sqrt{-g_{m n} \dot{x}^{m} \dot{x}^{n}\left(\Delta_{1}-2 \sqrt{\Delta_{2}} \frac{2 r|\dot{z}|}{\left|\dot{x}^{m} e_{m}{ }^{a} \xi_{a}\right|}\right)} .
$$

The Lagrangian is well defined if the admissible values of particle's velocity in the internal space $S^{2}$ are constrained on the manner

$$
\Delta_{1}>2 \sqrt{\Delta_{2}} \frac{2 r|\dot{z}|}{\left|\dot{x}^{m} e_{m} a \xi_{a}\right|}
$$

in addition to the causal restriction $g_{m n} \dot{x}^{m} \dot{x}^{n}<0$ in the space-time.

\section{Dynamical histories}

We finally turn to the analysis of dynamical histories in the model. To completely describe the mass shell, it is sufficient in fact to determine solutions of the dynamical equations with the minimal energy. The trajectories with $E>E_{o}$ can be simply restored from those with $E=E_{o}$ by applying all possible AdS-transformations.

The Hamiltonian action leading to the Lagrangian (77) reads

$$
S=\int \mathrm{d} \tau\left(p_{m} \dot{x}^{m}+\mathbf{k}_{a} \dot{\mathbf{b}}^{a}-\frac{1}{2} \sum_{I=5}^{8} \lambda_{I} T_{I}\right) .
$$

where $T_{5}=\mathbf{b}^{2}, T_{6}=(\mathbf{b}, \mathbf{k})$, and the constraints $T_{7}, T_{8}$ are given by Eqs. $(82,74,75)$. The corresponding equations of motion can be represented, with the aid of the constraints, in the form

$$
\begin{gathered}
\dot{x}^{m}-r^{2} \lambda_{7} \mathbf{p}^{m}-\Delta_{2} \lambda_{8} \frac{\mathbf{b}^{m}}{(\mathbf{b}, \mathbf{p})}=0 \\
\dot{\mathbf{p}}_{a}-\frac{1}{2} \mathcal{R}_{a b c d} \dot{x}^{m} e_{m}{ }^{b} M^{c d}=0
\end{gathered}
$$




$$
\begin{gathered}
\dot{\mathbf{b}}^{a}-\frac{1}{2} \lambda_{6} \mathbf{b}^{a}-r^{2} \lambda_{8}(\mathbf{b}, \mathbf{p})^{2} \mathbf{k}^{a}=0 \\
\dot{\mathbf{k}}^{a}+\lambda_{5} \mathbf{b}^{a}+\frac{1}{2} \lambda_{6} \mathbf{k}^{a}+\Delta_{2} \lambda_{8} \frac{\mathbf{p}^{a}}{(\mathbf{b}, \mathbf{p})}=0 .
\end{gathered}
$$

Here the covariant differentiation is defined by the rule $(78), \mathcal{R}_{a b c d}$ is the curvature tensor of the AdS space

$$
\mathcal{R}_{a b c d}=-\frac{1}{r^{2}}\left(\eta_{a c} \eta_{b d}-\eta_{a d} \eta_{b c}\right)
$$

The equations of motion should be supplemented by the global restriction on Lagrange multipliers

$$
\Delta_{1} \lambda_{7}>\Delta_{2} \lambda_{8}
$$

that guarantees the timelikeness of $\dot{x}^{m}$ at any point of the world line.

The above action possesses four gauge symmetries related to the constraints. Those associated with $T_{5}$ and $T_{6}$ can be fixed by imposing the following AdS-invariant gauge conditions

$$
\begin{gathered}
(\mathbf{p}, \mathbf{k})=0 \\
(\mathbf{p}, \mathbf{b})=-1
\end{gathered}
$$

Then on the mass shell, $\lambda_{5}$ and $\lambda_{6}$ are completely determined in the form

$$
\lambda_{5}=-\frac{\Delta_{2}}{r^{2}}\left(\lambda_{7}-\Delta_{1} \lambda_{8}\right) \quad \lambda_{6}=0
$$

Importantly enough, Eq. (97.a) implies

$$
\mathbf{k}^{0}=0
$$

for any dynamical history with the minimal energy.

Let us now choose a useful coordinate system $\left\{x^{m}\right\}$ on $M^{4}$ as follows

$$
\begin{gathered}
y^{5}=\rho \cos \left(x^{0} / r\right) \\
y^{0}=\rho \sin \left(x^{0} / r\right) \\
\vec{y}=\vec{x} .
\end{gathered}
$$

Here $0 \leq x^{0}<2 \pi r$ for $M^{4}$ and $-\infty<x^{0}<\infty$ for the universal covering space of $M^{4}$. We also set $\mathcal{G}(y)$ up as in Eq. (8). Then the vierbein reads

$$
e_{m}{ }^{a}=\left(\begin{array}{ccc}
\frac{\rho}{r} & \vdots & 0 \\
\cdot & \vdots & \ldots \ldots \ldots \ldots \\
0 & \vdots & \delta^{i j}-\frac{x^{i} x^{j}}{\rho(\rho+r)}
\end{array}\right) \quad i, j=1,2,3
$$


and the spin connection $w_{a b c}=e_{a}{ }^{m} w_{m b c}$ looks like

$$
\begin{gathered}
w_{0 i 0}=-w_{00 i}=\frac{x_{i}}{r \rho} \quad w_{i 0 j}=0 \\
w_{i j k}=\frac{1}{r(\rho+r)}\left(\delta_{i j} x_{k}-\delta_{i k} x_{j}\right)
\end{gathered}
$$

where $i, j, k=1,2,3$.

For the trajectories with the minimal energy, the equations of motion are drastically simplified. The relations $(52,98)$ and gauge conditions (97) imply that in this case the only time-dependent functions to be determined are $x_{0}(\tau), \vec{m}(\tau)$ and $\vec{n}(\tau)$, where

$$
\vec{m}=\frac{\vec{y}}{|\vec{y}|}=\frac{\overrightarrow{\mathbf{k}}}{|\overrightarrow{\mathbf{k}}|} \quad \vec{n}=\frac{\overrightarrow{\mathbf{p}}}{|\overrightarrow{\mathbf{p}}|}=\frac{\overrightarrow{\mathbf{b}}}{|\overrightarrow{\mathbf{b}}|}
$$

It is natural here to recall that the 3-vectors $\vec{m}, \vec{n}$ and $\vec{W}$ (64.b) form an orthonormal set at each moment of time evolution, $\vec{W}$ being time-independent. With the use of the expressions $(101,102)$, we derive from (95) the following equations

$$
\begin{gathered}
\frac{\dot{x}^{0}}{r}=E_{o}\left(\lambda_{7}-s^{2} \lambda_{8}\right) \\
\dot{\vec{m}}=s \vec{n}\left(\lambda_{7}-E_{o}^{2} \lambda_{8}\right) \\
\dot{\vec{n}}=-s \vec{m}\left(\lambda_{7}-E_{o}^{2} \lambda_{8}\right) .
\end{gathered}
$$

It follows from Eq. (96) that the r.h.s in (104) is strictly positive at each point of the world line

$$
\lambda_{7}-s^{2} \lambda_{8}>0 .
$$

Thus we are able to fix the gauge freedom associated with $T_{7}$ by imposing the gauge condition

$$
x^{0}=r \tau
$$

which relates the evolution parameter to the physical time. There is no physically preferable way to fix the gauge freedom related to $T_{8}$. The most elegant gauge condition seems to be

$$
\lambda_{8}=0
$$

Then on the mass shell, $\mathbf{b}$ is covariantly constant modulo local $\mathbf{b}$-rescalings, in accordance with (95.c), and strictly covariantly constant in the gauge (97). Choosing the gauge conditions $(107,108)$, the equations $(105)$ take the form

$$
\dot{\vec{m}}=\frac{s}{E_{o}} \vec{n} \quad \dot{\vec{n}}=-\frac{s}{E_{o}} \vec{m} .
$$

In this gauge, the trajectories for $s \neq 0$ are in general globally defined only on the universal covering space of $M^{4}$. The same feature is known for the on-shell fields describing free particles with spin on the AdS space $[9,13]$. 


\section{Conclusion}

Let us give a brief overview of the results and some comments. In this paper we have suggested the model for a spinning particle on $d$-dimensional anti-de Sitter space as a mechanical system with the configuration space $\mathcal{M}^{2(d-1)}=M^{d} \times S^{d-2}$. In any space-time dimensions, the model possesses two gauge symmetries. Their origion lies in the fact that the phase-space counterparts of the second- and fourth-order Casimir operators of $s o(d-1,2)$ are constrained to have constant values $\Delta_{1}, \Delta_{2}$ on the phase space.

We have thoroughly studied the model in four dimensions. For $\Delta_{2}=0<\Delta_{1}$, our model is equivalent to the theory of a massive spinless particle (38). The case of massive spinning particles is specified by the condition $0<2 \sqrt{\Delta_{2}}<\Delta_{1}$. Only under the choice $0 \leq 2 \sqrt{\Delta_{2}}<\Delta_{1}$, the energy proves to have a global positive minimum $E_{o}$ over the phase space, $E_{o}$ given by Eq. (58). The last condition appears to be equivalent to the relation $E_{o}>s \geq 0$, where $s$ (58) is the value of the total angular momentum in any state with the minimal energy. Thus, $s$ can be naturally identified with the spin of the particle. It is worth noting that the inequality $E_{o}>s$ has been postulated in Ref. [20] to be the classical analogue of the quantum massive condition $E_{o}>s+1$.

In summary, in $d=4$ and for the restricted set of parameters $0 \leq 2 \sqrt{\Delta_{2}}<\Delta_{1}$, our model can be conceptually treated as a minimal and unified model of a massive particle with fixed, but arbitrary, spin on the AdS space. The model is minimal, because its configuration manifold has minimally possible dimension to describe the evolution in space-time and spin dynamics. It is unified, since the configuration space is spin-independent; the dynamics is specified by the choice of parameters $\Delta_{1}$, $\Delta_{2}$ entering the Lagrangian.

When $2 \sqrt{\Delta_{2}}=\Delta_{1}$, the energy remains positive over the phase space, but has no global minimum and can approach zero in some limiting directions. This choice of the parameters should correspond to massless particles. However, a simple counting shows that for $\Delta_{2}>0$ the model has too many degrees of freedom to describe a true massless dynamics. It is still unclear whether there exists a universal configuration space for massless spinning particles or not.

As we have demonstrated in sec. 6, the dynamical equations of the model can be readily integrated after specifying simple gauge conditions. But since the original equations of motion (95) involve arbitrary functions $\lambda_{5}, \ldots, \lambda_{8}$, which get fixed only upon putting gauge conditions up, the explicit structure of dynamical trajectories is gauge dependent and hence has no direct physical interest. Among gauge invariant properties of the model are the conditions (61) that characterize the states with the minimal energy. Eq. (61.a) leads to a rather unusual effect, from the point of view of our flat-space intuition. It is seen that when $E_{o}$ approaches its lower bounbary, $E_{o} \approx s$, the particle with $E=E_{o}$ turns out to be located far away from the origion

$\vec{y}=0$. Nevertheless, this result is very natural for the AdS universe and can be explained similar to what have been said (see, for example, [11]) to demonstrate 
the statement that singleton are physically unobservable. Indeed, let us pass from dimensionless units for energy to standard ones by redefining $E \rightarrow r^{-1} E$. Then the relation $E_{o} \approx s$ turns into energy $=r^{-1} \times$ angular momentum,$r^{-2}$ being proportional to the curvature of the AdS space and hence very small. Therefore, the particle moves at distances of the same order as the "radius" of the AdS space.

Finally, let us shortly comment on quantization of the model. It has been argued, in sec. 4, that every physical observable in the model is a function of the Hamilton generators of $s o(3,2)$. So, the covariant operatorial quantization of the model reduces to constructing unitary, positive-energy representatios of $s o(3,2)$, what has been worked out in Ref. [10]. A non-trivial question, however, is how to construct a coordinate realization for this (constrained) quantum mechanics, i.e. to realize the massive, positive-energy AdS-representations in some function spaces over $\mathcal{M}^{6}$ with an apropriate Hilbert space structure. In the case of flat space-time, the analogous problem has been exhaustively studied in our previous paper [6] where all such realizations were classified. We intend to give a similar consideration for the AdS case in a forthcoming publication [21].

\section{Acknowledgements}

It is a pleasure to thank N. Dragon, O. Lechtenfeld and A. Zubarev for useful discussions. This work was supported in part by European Community grant No. INTAS93-2058 and by the International Science Foundation grant No. M21000. The work of SMK was supported in part by the Alexander von Humboldt Foundation. The work of SLL was partly supported by the Royal Society under a Kapitza Fellowship programme.

\section{References}

[1] D.P. Sorokin, V.I. Tkach and D.V. Volkov, Mod. Phys. Lett. A4, 901 (1989); D.P. Sorokin, V.I. Tkach, D.V. Volkov and A.A. Zheltukhin, Phys. Lett. B216, 302 (1989).

[2] A.S. Galperin, P.S. Howe and K.S. Stelle, Nucl. Phys. B368, 248 (1992); A. Galperin and E. Sokatchev, Phys. Rev. D46, 714 (1992).

[3] F. Delduc, A. Galperin, P. Howe and E. Sokatchev, Phys. Rev. D47, 578 (1993).

[4] I.M. Gel'fand, R.A. Milnos and Z.Ya. Shapiro, Representations of the Rotation and Lorentz Group and Their Applications (Pergamon, London, 1963).

[5] R. Penrose and W. Rindler, Spinors and Space-time (Cambridge Univ. Press, Cambridge, 1986).

[6] S.M. Kuzenko, S.L. Lyakhovich and A.Yu. Segal, Int. J. Mod. Phys. A10, 1529 (1995). 
[7] S.M. Kuzenko, S.L. Lyakhovich and A.Yu. Segal, Phys. Lett. B348, 421 (1995).

[8] S.W. Hawking and G.F.R. Ellis, The Large Scale Structure of Space-time (Cambridge Univ. Press, Cambridge, 1973).

[9] C. Fronsdal, Rev. Mod. Phys. 37, 221 (1965); Phys. Rev. D10, 589 (1974); C. Fronsdal and R.B. Haugen, Phys. Rev. D12, 3810 (1975); C. Fronsdal, Phys. Rev. D12, 3819 (1975).

[10] M.T. Evans, J. Math. Phys. 8, 170 (1967).

[11] A. Angelopoulos, M. Flato, C. Fronsdal and D. Sternheimer, Phys. Rev. D23, 1278 (1981).

[12] P.A.M. Dirac, J. Math. Phys. 4, 901 (1963).

[13] C. Fronsdal, Phys. Rev. D20, 848 (1979); J. Fang and C. Fronsdal, Phys. Rev. D22, 1361 (1980).

[14] B.W. Keck, J. Phys. A8, 1819 (1975).

[15] B. Zumino, Nucl. Phys. B127, 189 (1977).

[16] S.J. Gates, M.T. Grisaru, M. Roček and W. Siegel, Superspace (BenjaminCummings, Reading, MA, 1983).

[17] I.L. Buchbinder and S.M. Kuzenko, Ideas and Methods of Supersymetry and Supergravity (Institute of Physics Publishing, Bristol and Philadelphia, 1995).

[18] E.S. Fradkin and M.A. Vasiliev, Nucl. Phys. B291, 41 (1987); Ann. Phys. (N.Y.) 177, 63 (1987).

[19] M.A. Vasiliev, Ann. Phys. (N.Y.) 190, 59 (1989); Phys. Lett. B243, 378 (1990); Clas. Quantum Grav 8, 1387 (1991).

[20] A.M. El Gradechi and S. De Bievre, Ann. Phys. (N.Y.) 235, 1 (1994).

[21] S.M. Kuzenko, S.L. Lyakhovich, A.Yu. Segal and A.A.Sharapov, in preparation.

[22] J. Wess and J. Bagger, Supersymmetry and Supergravity (Cambridge Univ. Press, Cambridge, 1983). 